\title{
Improved Durability of Solvent Impregnated Resin Using Acidic Organophosphorus Extractant
}

\author{
Chaitanya Raj ADHIKARI, Yumiko SATO and Mikiya TANAKA* \\ National Institute of Advanced Industrial Science and Technology (AIST), Japan
}

\begin{abstract}
Solvent impregnated resins (SIRs) are an effective means for the separation of metal ions from their mixtures. However, their durability is limited due to dissolution of the extractant from the resin. Our previous studies on the repeated column test using PC88A-SIR in order to remove zinc from the model electroless nickel plating bath showed that zinc adsorption capacity gradually decreased over a few cycles. In this paper, the distribution of PC88A and Cyanex 272 between the aqueous solution and SIR or blank resin was measured batchwise. Then the column experiment was carried out, and the following two methods have been found to be effective: (i) Post-column process in which the feed solution is passed through the PC88A-SIR and then the equal weight of the blank resin (Amberlite XAD7HP) and (ii) replacement of the extractant by weaker acid, Cyanex 272. In the latter, the zinc adsorption ability of the SIR was constant for at least 37 cycles. It is suggested that the combination of these two methods significantly improves the SIR durability.
\end{abstract}

Key words: Solvent impregnated resin, Solvent extraction, Zinc, PC88A, Cyanex 272

\section{Introduction}

Among the various conventional methods to separate metal ions from multi-component solutions, solvent extraction and ion exchange resins (including chelating resins) are often employed in industrial processes. Each of the techniques has its own set of advantages and disadvantages. However, an alternative technique combining the merits of both techniques has been developed by Warshawsky in the form of solvent-impregnated resins (SIRs) in which macroporous, polymeric resins with high surface areas are physically impregnated with an organic extractant (complexant) ${ }^{1}$. Due to their easy preparation procedure, simplicity of use, and their versatility to meet the specific requirement of metal separation, the SIRs are considered to be a very effective means to separate metal ions from the multi-component mixtures. A large number of pa-

Paper presented at the $11^{\text {th }}$ Japan/Korea International Symposium on Resources Recycling and Materials Science, 17-19 June 2013, Osaka, Japan, and also presented at the $12^{\text {th }}$ International Symposium on East Asian Resources Recycling Technology, 3-5 Nov. 2013, Zhangjiajie, P.R. China

Accepted 17 January, 2014

*e-mail: mky-tanaka@aist.go.jp pers related to their application for the separation and recovery of target metal ions is available in the literature ${ }^{2-15}$.

However, a major drawback of the SIRs is their limited durability. A gradual loss in the capacity of the SIRs to adsorb the target metal ions has been observed due to the leakage of the extractant from the resin into the aqueous phase ${ }^{9,10}$. We also reported in our previous study using PC88A-SIR that the zinc adsorption capacity of the SIR at the $15^{\text {th }}$ cycle of a repeated column test was almost half that of the initial cycle ${ }^{14}$. Limited efforts have been made in the past in order to improve the durability of the SIR for metal adsorption. According to the literature, a major attempt was to form a semi-permeable membrane on the outer surface of the resin to inhibit the leakage of the extractant into the aqueous phase. Although such a coating process has been successful to some extent ${ }^{8-10,13,15}$, the preparation procedure of SIR becomes less simple.

In this paper, we describe our efforts to improve the durability of an SIR using acidic organophosphorus extractants for the zinc removal from the electroless nickel plating bath without the coating process. After batch dissolution and adsorption measurements of the extractants, two 
kinds of attempts were made: (i) placement of the blank resin after the SIR column, and (ii) use of weaker acid as the extractant.

In these attempts, we measure the zinc adsorption from a model electroless nickel plating bath because of our concern with the bath life extension. As described in our previous papers ${ }^{14,16}$, a gradual accumulation of zinc in the plating bath as a byproduct of the pre-plating process lowers the rate and quality of the plating, thus leading to the shorter bath life.

\section{Experimental}

\subsection{Reagents and chemicals}

Amberlite XAD7HP (Rohm and Haas) used as the SIR substrate was a highly porous (porosity $\geq 0.50 \mathrm{~mL} / \mathrm{mL}$ ) industrial grade polymeric adsorbent in the form of white beads (surface area $\geq 380 \mathrm{~m}^{2} / \mathrm{g}$, specific gravity $\left.=1.06-1.08\right)^{17}$. It was pretreated by washing three times with methanol followed by drying at $80^{\circ} \mathrm{C}$ for two hours. PC88A was a Daihachi product (active component $=$ 2-ethylhexylphosphonic acid mono-2-ethylhexyl ester, specific gravity $=0.952 \pm 0.015$ at $\left.20^{\circ} \mathrm{C}\right)^{18}$. Cyanex 272 was a Cytec product (active component $=$ bis (2,4,4-trimethylpentyl)phosphinic acid, specific gravity $=0.92$ at $\left.24^{\circ} \mathrm{C}\right)^{19}$. Both PC88A and Cyanex 272 were used without further purification for the preparation of the SIRs. All other chemicals used in this experiment were of reagent grade.

\subsection{Preparation of the SIRs and the model electroless nickel plating bath}

PC88A-SIR or Cyanex 272-SIR were prepared by the dry method ${ }^{14}$. The extractant content will be expressed as its weight percent in the SIR. The solution used for the repeated column test was a model electroless nickel plating bath containing $4.4 \mathrm{~g} / \mathrm{L}$ nickel and $0.08 \mathrm{~g} / \mathrm{L}$ zinc at $\mathrm{pH} 4.7$, prepared by dissolving the specified amounts of sodium sulfate $\left(\mathrm{Na}_{2} \mathrm{SO}_{4}\right)$, zinc sulfate $\left(\mathrm{ZnSO}_{4}\right)$, nickel sulfate hexahydrate $\left(\mathrm{NiSO}_{4} \cdot 6 \mathrm{H}_{2} \mathrm{O}\right)$, sodium phosphinate monohydrate $\left(\mathrm{NaH}_{2} \mathrm{PO}_{2} \cdot \mathrm{H}_{2} \mathrm{O}\right)$, disodium hydrogen phosphate pentahydrate $\left(\mathrm{Na}_{2} \mathrm{HPO}_{3} \cdot 5 \mathrm{H}_{2} \mathrm{O}\right)$ as well as sulfuric and lactic acids ${ }^{14}$.

\subsection{Batch experiment}

To examine the dissolution of the extractant from the SIR, aqueous solutions of $0.1 \mathrm{~mol} / \mathrm{L}$ citric acid and $0.1 \mathrm{~mol} / \mathrm{L}$ sodium citrate were separately prepared and mixed at suitable proportions to prepare different $\mathrm{pH}$ solutions $(\mathrm{pH} 4-6)$. After horizontally mixing the SIR $(100 \mathrm{mg})$ with the solution $(15 \mathrm{~mL})$ at $140 \mathrm{rpm}$ for $24 \mathrm{~h}$ in a water bath at $25^{\circ} \mathrm{C}$, the amount of phosphorous in the solution was analyzed by ICP-AES (Shimadzu ICPS-7500). The reverse experiment, i.e., the adsorption of the extractant by the fresh resin from the aqueous raffinate during the dissolution test was also conducted at the same solid-liquid ratio.

\subsection{Column operation}

For the repeated column operation (durability test), a glass column $(11 \mathrm{~mm}$ in inner diameter and $15 \mathrm{~cm}$ in height) was packed with the SIR $(1 \mathrm{~g})$. A model electroless nickel plating bath was passed upward through it at a fixed flow rate (25 or $27 \mathrm{~mL} / \mathrm{h}$ ) using a peristaltic pump. The temperature of the column was maintained at $25^{\circ} \mathrm{C}$ by a water jacket. The effluent was collected in a beaker during each cycle and analyzed by AAS (Perkin-Elmer AAnalyst 100). The adsorbed metal ions were eluted with $2 \mathrm{~mol} / \mathrm{L} \mathrm{H}_{2} \mathrm{SO}_{4}$ at the same flow rate in order to make the column ready for the next cycle. The content of the extractant in the SIR was determined by nonaqueous neutralization titration after dissolving the extractant into ethyl alcohol.

\section{Results and Discussion}

\subsection{Batch studies}

\subsubsection{Dissolution of the extractant}

The effects of $\mathrm{pH}$, kind of the extractant, and the presence of zinc on the dissolution of the extractant in the SIR into the citrate solution were batchwise measured as shown in Fig. 1. Here, the extractant dissolution (\%) is defined as the percentage of dissolved phosphorous weight to the initial phosphorous weight in the SIR, and the $\mathrm{pH}$ is the initial value. Because the aqueous solution has buffering ability, the difference in the initial and final $\mathrm{pH}$ values is less than 0.2 . Zinc adsorption efficiencies are thought to be more than $90 \%$ in the present $\mathrm{pH}$ range ${ }^{14}$. The results show that (i) the extractant dissolution was enhanced at the $\mathrm{pH}$ higher than 5, (ii) the dissolution of Cyanex 272 is less than that of PC88A, and (iii) the presence of zinc in the aqueous solution clearly decreases the extractant dissolution.

The mechanism of the dissolution of acidic extractants in SIRs to the aqueous solution would be similar to that in solvent extraction systems ${ }^{20}$. The dissolution equilibrium of the extractant, $\mathrm{H}_{2} \mathrm{~L}_{2}$, is expressed as

$$
\overline{\mathrm{H}_{2} \mathrm{~L}_{2}} \leftrightarrow 2 \mathrm{HL}
$$

where the overbar denotes the species in the SIR phase. The distribution equilibrium constant, $K_{\mathrm{d}}$, 
Improved Durability of Solvent Impregnated Resin Using Acidic Organophosphorus Extractant

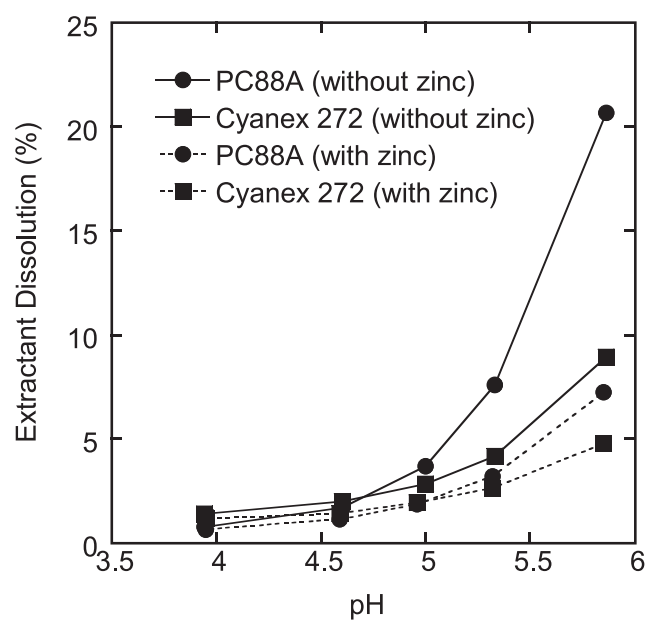

Fig. 1 Extractant dissolution as a function of $\mathrm{pH}$. (Conditions: Weight of the resin $(30 \%$ PC88A-SIR or $30 \%$ Cyanex 272-SIR) $=100 \mathrm{mg}$, volume of aqueous solution $=15 \mathrm{~mL}$, concentration of zinc in the solution (in the case of its presence): $50 \mathrm{mg} / \mathrm{L}$ )

is written as

$$
K_{\mathrm{d}}=[\mathrm{HL}]^{2} / m\left(\overline{\mathrm{H}_{2} \mathrm{~L}_{2}}\right)
$$

where the brackets [ ] and $m$ denote the molar concentration in the aqueous solution and the content in the SIR, respectively. When the $\mathrm{pH}$ becomes higher, the dissolved HL dissociates into hydrogen ion and the extractant anion as

$$
\mathrm{HL} \leftrightarrow \mathrm{H}^{+}+\mathrm{L}^{-}
$$

The acid dissociation constant, $K_{\mathrm{a}}$, is expressed as

$$
K_{\mathrm{a}}=\left[\mathrm{H}^{+}\right]\left[\mathrm{L}^{-}\right] /[\mathrm{HL}]
$$

Thus, the total aqueous HL concentration, $[\mathrm{HL}]_{\mathrm{T}}$, is written as

$$
[\mathrm{HL}]_{\mathrm{T}}=K_{\mathrm{d}}^{1 / 2} m\left(\overline{\mathrm{H}_{2} \mathrm{~L}_{2}}\right)^{1 / 2}\left(1+K_{\mathrm{a}} /\left[\mathrm{H}^{+}\right]\right)
$$

This equation indicates that (i) $[\mathrm{HL}]_{\mathrm{T}}$ does not depend on $\mathrm{pH}$ when the $\mathrm{pH}$ is sufficiently low and (ii) $[\mathrm{HL}]_{\mathrm{T}}$ increases with $\mathrm{pH}$ when the $\mathrm{pH}$ becomes higher.

This model explains the results (i)-(iii): the general behavior of the extractant dissolution without zinc as a function of $\mathrm{pH}$ is in agreement with the result (i). Because Cyanex 272 is weaker acid than PC88A (the $\mathrm{pKa}\left(=-\log K_{\mathrm{a}}\right)$ values of these reagents in $75 \%$ ethyl alcohol are reported to be 4.10 for PC88A and 5.05 for Cyanex $272^{21}$ ), the $\mathrm{pH}$ in which the dissolution starts to increase is higher for Cyanex 272 than for PC88A, which verifies the result (ii). Hydrohobicity of the zincextractant complex is likely to be higher than that of the extractant, which explains the result (iii).

\subsubsection{Re-adsorption of the extractant into the blank resin}

The raffinate of the dissolution experiment using PC88A-SIR at the initial $\mathrm{pH}$ of 5.86 was contacted with the fresh XAD7HP (blank resin). The result shows that $96 \%$ of the dissolved PC88A was absorbed.

\subsection{Column studies}

\subsubsection{Post-column process}

From the results described in section 3.1.2, it is expected that if the effluent discharged from the SIR contacts with the blank resin, the extractant in the effluent is re-adsorbed into the blank resin. Therefore, this post-column process would reduce the extractant loss and, at the same time, maintain the metal adsorption ability because the fresh blank resin would be gradually converted to an SIR. Thus, a specified weight of XAD7HP was placed on the PC88A-SIR ( $1 \mathrm{~g})$ in the column, and a durability test was carried out using the model bath. The results are shown in Fig. 2 together with the smoothed curve of our previous data obtained without the blank resin ${ }^{14}$. This figure demonstrates that an equal weight of the blank resin added to the SIR appreciably improved the durability of the column while the amount of the blank resin decreased to one-fourth had no

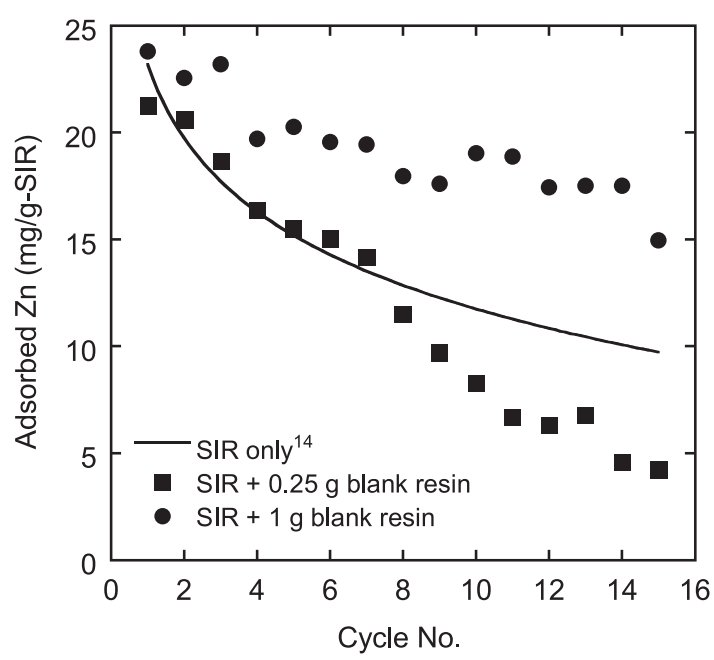

Fig. 2 Adsorbed zinc as a function of the number of repetition using the post-column of blank resin after $30 \%$ PC88A-SIR. (Conditions: Weight of $30 \%$ $\mathrm{PC} 88 \mathrm{~A}-\mathrm{SIR}=1 \mathrm{~g}$, volume of the solution $=500$ $\mathrm{mL}$, flow rate $=27 \mathrm{~mL} / \mathrm{h}$ ) 
Table 1 PC88A contents before and after the durability test

\begin{tabular}{lcccccc}
\hline & & \multicolumn{2}{c}{ Before the test } & & \multicolumn{2}{c}{ After the test } \\
\cline { 3 - 4 } \cline { 6 - 7 } & $\begin{array}{l}\text { Sec- } \\
\text { tion }\end{array}$ & $\begin{array}{c}\text { Weight } \\
(\mathrm{g})\end{array}$ & $\begin{array}{c}\text { PC88A } \\
\text { content } \\
(\%)\end{array}$ & & $\begin{array}{c}\text { Weight } \\
(\mathrm{g})\end{array}$ & $\begin{array}{c}\text { PC88A } \\
\text { content } \\
(\%)\end{array}$ \\
\hline Post- & 1 & 0.333 & 0 & & 0.352 & 5.2 \\
column & 2 & 0.333 & 0 & & 0.359 & 7.3 \\
& 3 & 0.333 & 0 & & 0.378 & 11.7 \\
\hline Main & 4 & 0.500 & 30.8 & & 0.502 & 31.2 \\
column & 5 & 0.500 & 30.8 & & 0.460 & 24.8 \\
\hline
\end{tabular}

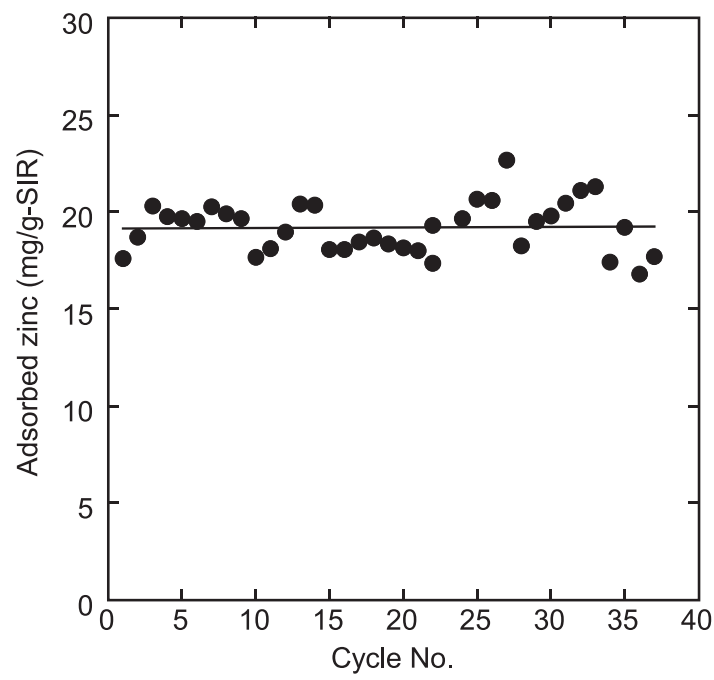

Fig. 3 Adsorbed zinc as a function of the number of repetition using 52\% Cyanex 272-SIR (Conditions: Weight of the resin: $1 \mathrm{~g}$, volume of the solution $($ model bath $)=1000 \mathrm{~mL}$, flow rate: $25 \mathrm{~mL} / \mathrm{h})$

effect.

After the durability test using the column containing $1 \mathrm{~g}$ of the blank resin, the column was divided into five horizontal sections in order to determine the PC88A content in each section. The result was shown in Table 1, indicating that the blank resin captured PC88A dissolved from the main column.

\subsubsection{Introduction of extractant with higher $\mathbf{p K}_{\mathrm{a}}$}

Fig. 1 strongly suggests that the extractant loss is reduced by replacing PC88A to Cyanex 272 particularly when the feed solution $\mathrm{pH}$ is in the range of 4-6. Thus, Cyanex 272-SIR (52\%) was packed into the column and the durability test was carried out with the results shown in Fig. 3. The amount of zinc adsorbed by the Cyanex 272-SIR packed column is almost constant over 37 cycles; that is, there is no decrease in the capacity of the SIR for zinc adsorption. This is the longest cycling of the SIR reported so far.

\section{Conclusions}

In order to improve the durability of the SIR using an acidic organophosphorus extractant for zinc removal in the electroless nickel plating bath, two kinds of attempts have been made after the initial distribution measurement of the extractants between the aqueous solution and the resin. The first one is the post-column process placing a blank resin bed after the SIR bed. Clear improvement of the durability was seen although the zinc adsorption capacity still slightly decreased with the repeated use. The second one is to replace the extractant by that with a higher $\mathrm{p} K_{\mathrm{a}}$. When the PC88A was replaced by a weaker acid, Cyanex 272, the durability was appreciably improved; the zinc adsorption capacity is constant for at least 37 cycles. If these two methods are combined, the SIR would become more practical without a coating process.

\section{Acknowledgement}

The authors express their sincere thanks to the New Energy and Industrial Technology Development Organization (NEDO) for its financial support and Ms. Takayo Harada for her experimental assistance.

\section{References}

1. A. Warshawsky: In Ion Exchange and Solvent Extraction, A Series of Advances (J.A. Marinsky, Y. Marcus, Eds.), Marcel Dekker (New York), Vol. 8, pp. 234-259 (1981)

2. Y. Wakui, H. Matsunaga, T.M. Suzuki: Anal. Sci., 4, pp. 325-327 (1988)

3. J.L. Cortina, N. Miralles, A. Sastre, M. Aguilar, A. Profumo, M. Pesavento: React. Polym., 18, pp. $67-75(1992)$

4. R.S. Juang and J.Y. Su: Ind. Eng. Chem. Res., 31, pp. 2779-2783 (1992)

5. J.L Cortina, N. Miralles, A. Sastre, M. Aguilar, A. Profumo, M. Pesavento: React. Polym., 21, pp. 89-101 (1993)

6. J. Shibata, T. Nagasawa, M. Mashimo: Shigen-toSozai, 110, pp. 191-194 (1994)

7. N. Kabay, M. Demircioglu, H. Ekinci, M. Yuksel, M. Saglam, M. Streat: React. Funct. Polym., 38, pp. 219-226 (1998)

8. D. Muraviev, L. Ghantous, M. Valiente: React. Funct. Polym., 38, pp. 259-268 (1998)

9. S.D. Alexandratos and K.P. Ripperger: Ind. Eng. Chem. Res., 37, pp. 4756-4760 (1998)

10. A.W. Trochimczuk, N. Kabay, M. Arda, M. Streat: React. Funct. Polym., 59, pp. 1-7 (2004)

11. C.Y. Shiau, C.L. Lin, H.S. Chang: Ind. Eng. Chem. 
Res., 44, pp. 4771-4777 (2005)

12. R. Navarro, V. Gallardo, I. Saucedo, E. Guibal: Hydrometallurgy, 98, pp. 257-266 (2009)

13. Y. Yuan, J. Liu, B. Zhou, S. Yao, H. Li, W. Xu: Hydrometallurgy, 101, pp. 148-155 (2010)

14. C.R. Adhikari, H. Kumano, M. Tanaka: Solvent Extr. Ion Exch., 29, pp. 323-336 (2011)

15. S. Nishihama, K. Kohata, K. Yoshizuka: Sep. Purif. Technol., 118, pp. 511-518 (2013)

16. M. Tanaka, Y. Saiki, K. Hagisawa, H. Narita: Proceedings of the International Solvent Extraction Conference (ISEC), Beijing, pp. 1374-1377 (2005)

17. Rohm and Haas Company: Amberlite XAD-7HP, Product data sheet (2003 Oct.)
18. Daihachi Chemical Industry Co., Ltd.: Product information on metal extractants, URL: www. daihachi-chem.co.jp (accessed on June 10, 2010)

19. Cytech Industries Inc.: Product information on solvent extraction reagent, Cyanex 272, URL: www.cytech.com /speciality chemicals (accessed on January 22, 2013)

20. K. Mimura, J. Shibata, M. Sano, S. Nishimura: Technology Report of Kansai University, 27, pp. 91-100 (1986)

21. M. Cox, M. Elizalde, J. Castresana, N. Miralles: Proceedings of the International Solvent Extraction Conference (ISEC'83), Denver, CO, pp. 268269 (1983) 